\title{
EFFECTIVNESS OF TARGET ANTIMICROBIAL THERAPY OF SEVERE CHRONIC PERIODONTITIS PART III: CLINICAL ATTACHMENT GAIN
}

\author{
Kotsilkov K., Chr. Popova \\ Department of Periodontology, Faculty of Dental Medicine, \\ Medical University - Sofia, Bulgaria
}

\begin{abstract}
:
The main goal of the periodontal therapy is the regeneration of the affected periodontal structures and achievement of stable periodontal attachment. The contemporary statement regarding the effectiveness of the periodontal therapy is that healing results with more coronal level of the connective tissue attachment are associated with better long term stability.

Aim: Evaluation of the effectiveness of treatment of severe chronic periodontitis with additional target antibiotic administration in comparison with the therapy with adjunctive antimicrobial combination amoxicillin + metronidazole and conventional mechanical periodontal treatment regarding the mean clinical attachment gain, reduction of the attachment loss extent and the variations of the attachment level in sites with initial CAL from $1-2 \mathrm{~mm}$, from $3-4 \mathrm{~mm}$ and above $5 \mathrm{~mm}$.

Results: In all study groups mean clinical attachment gain has been achieved after the non-surgical periodontal therapy. In the group with target antibiotic administration the highest reduction of periodontal sites with CAL above $5 \mathrm{~mm}$ is reported.
\end{abstract}

Key words: periodontitis, adjunctive antibiotic therapy, target antibiotic administration, clinical attachment level gain, long-term maintenance.

\section{BACKGROUND:}

The microbial aetiology of inflammatory periodontal diseases provides the rationale for the use of antimicrobial medication in periodontal therapy. The evidence for bacterial specificity in periodontitis has accumulated and strengthened over the past three decades. The concept of bacterial specificity and data that antibiotic agent in vivo can exceed concentrations necessary to kill or inhibit the pathogen(s) advocate the contemporary approach of adjunctive antimicrobial periodontal treatment $(2,5,6,7,8,10,11)$.

The variety in the composition of the subgingival pathogenic flora and the specific sensitivity of the periodontopathogens predetermines the necessity from microbiological identification prior the antimicrobial treatment. The composition of the residual subgingival flora could predetermine the prognosis of the periodontal disease and the modalities of the supportive periodontal treatment $(12,13,14)$.

The main goal of the periodontal therapy is the regeneration of the affected periodontal structures and achievement of stable periodontal attachment. The contemporary statement regarding the effectiveness of the periodontal therapy is that healing results with more coronal level of the connective tissue attachment are associated with better long term stability. Therefore the gain of clinical attachment level is accepted as the primary criterion in the evaluation of the effectiveness of the non-surgical periodontal therapy $(1,3,4,9,15)$.

\section{AIM:}

Evaluation of the effectiveness of treatment of severe chronic periodontitis with additional target antibiotic administration in comparison with the therapy with adjunctive antimicrobial combination amoxicillin + metronidazole and conventional mechanical periodontal treatment regarding the mean clinical attachment gain, reduction of the attachment loss extent and the variations of the attachment level in sites with initial CAL from $1-2 \mathrm{~mm}$, from $3-4 \mathrm{~mm}$ and above $5 \mathrm{~mm}$.

\section{MATERIALS AND METHODS:}

Patients with active periodontal disease with 3468 affected sites were recruited in this study. Inclusion criteria were at least 20 teeth, 6 sites with $\mathrm{PD}>5 \mathrm{~mm}, 2$ sites with $\mathrm{PD}>7 \mathrm{~mm}$ in different dentition quadrants. Exclusion criteria were pregnancy, lactation, systemic antibiotic treatment in the previous 3 months. The patients were divided in tree groups:

- Test group 1 (TG1) - patents with 834 affected sites - conventional mechanical periodontal therapy and adjunctive broad spectrum systemic antibiotic treatment (Amoxicillin $500 \mathrm{mg} /$ Metronidazol 250mg - tid for 10 days.

- Test group 2 (TG2) - patents with 1110 affected sites - conventional mechanical periodontal therapy and adjunctive specific systemic antibiotic administration after microbiological testing for susceptibility.

- Control group (CG) - patients with 1524 affected sites - conventional mechanical periodontal therapy only. 


\section{Clinical parameters:}

A full range of periodontal diagnostic measurements were registered before and after treatment. The collected data includes: Hygiene Index (HI), Gingival bleeding index (GBI), Probing depth (PD), Clinical attachment level (CAL), Gingival recession (R), Furcation involvement (F) and Mobility (M) were carried out all patients. The measurements were registered on six points on every tooth (mesiobuccal, buccal, distobuccal, distolingual, lingual, mesiolingual) with Williams's periodontal probe. The alveolar bone loss was registered on panoramic, segment and bite-wing x-rays.

\section{RESULTS:}

The achieved gain of clinical attachment is the index of the highest importance regarding the efficiency of the periodontal therapy because the gain of attachment indicates a better healing result compared to the reduction of the probing depth. The reduction of the periodontal pockets is a

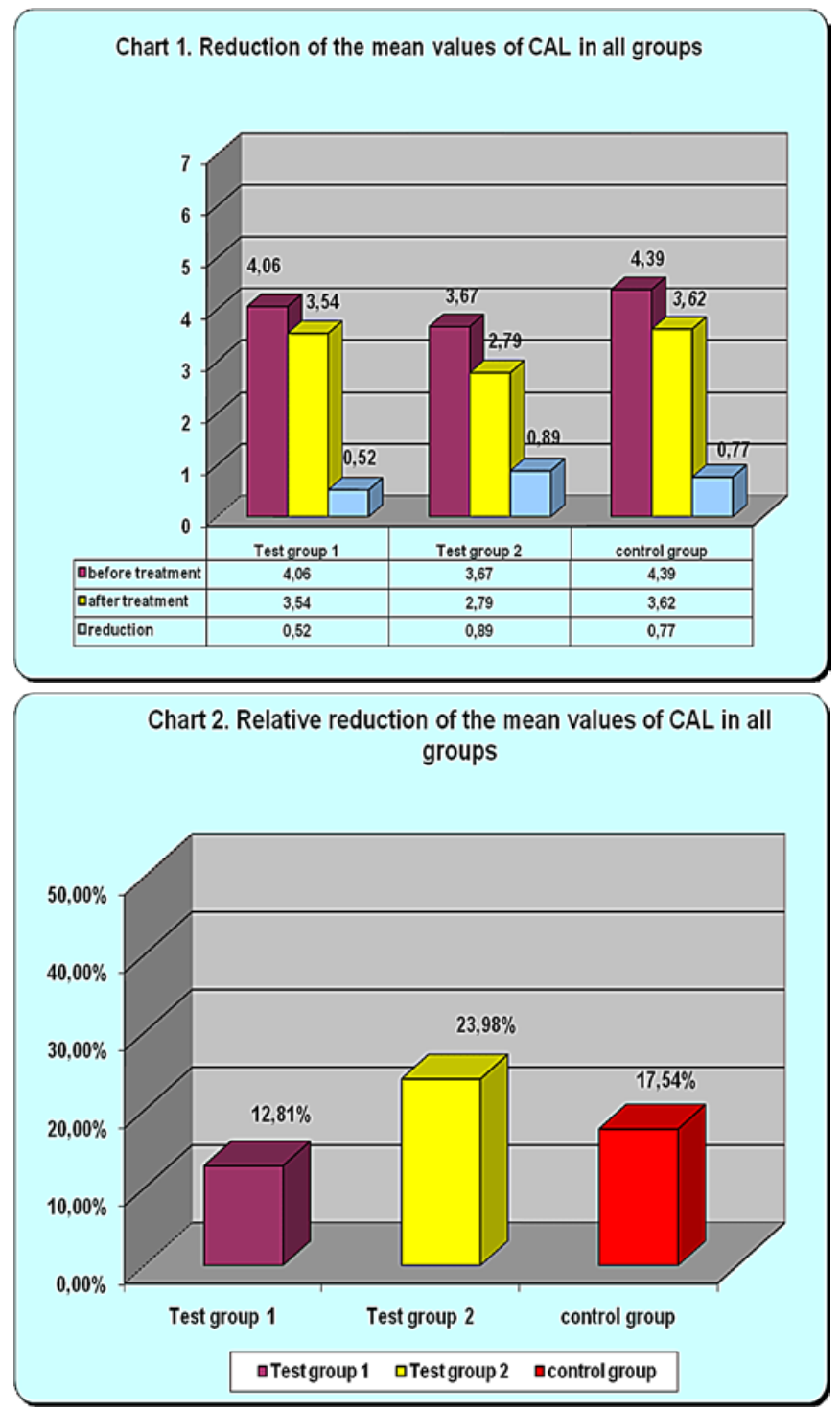

result basically from the healing process in the gingiva while the attachment gain represents a coronal migration of the periodontal support.

The collected data on the reevaluation after the periodontal therapy suggest that the target antimicrobial administration could enhance the healing in the periodontal pockets thus improving the clinical attachment gain. The higher degree of elimination of the periodontal pathogens with the target antimicrobial approach provides a better condition in the periodontal environment needed for the attaining of the new periodontal attachment.

In all study groups mean clinical attachment gain has been achieved after the non-surgical periodontal therapy. In the group with target antibiotic administration the highest reduction of periodontal sites with CAL above $5 \mathrm{~mm}$ is reported. These results advocate the effectiveness of the target adjunctive antimicrobial treatment in order to achieve better prerequisite for the future maintenance of the periodontal health.

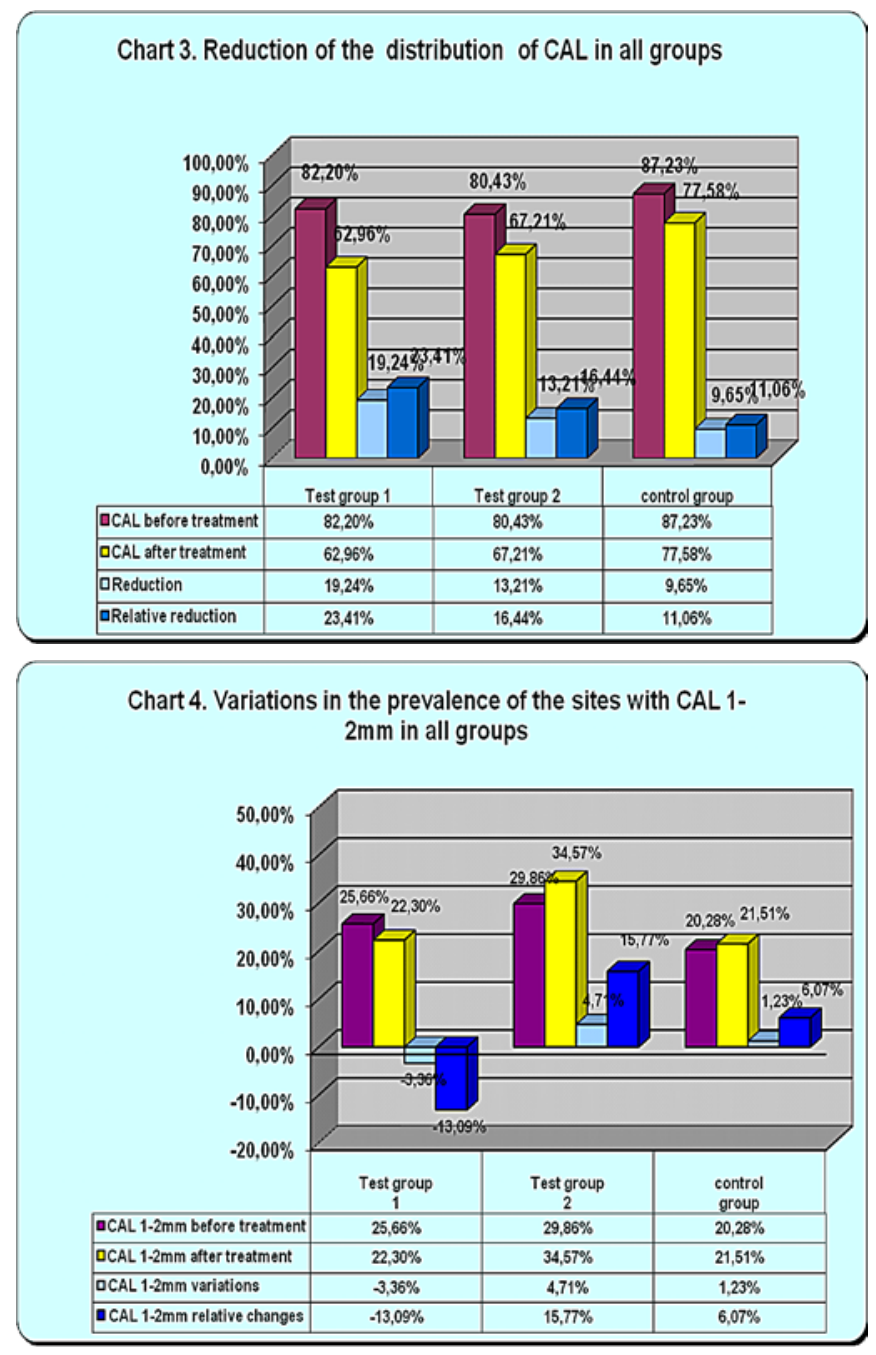



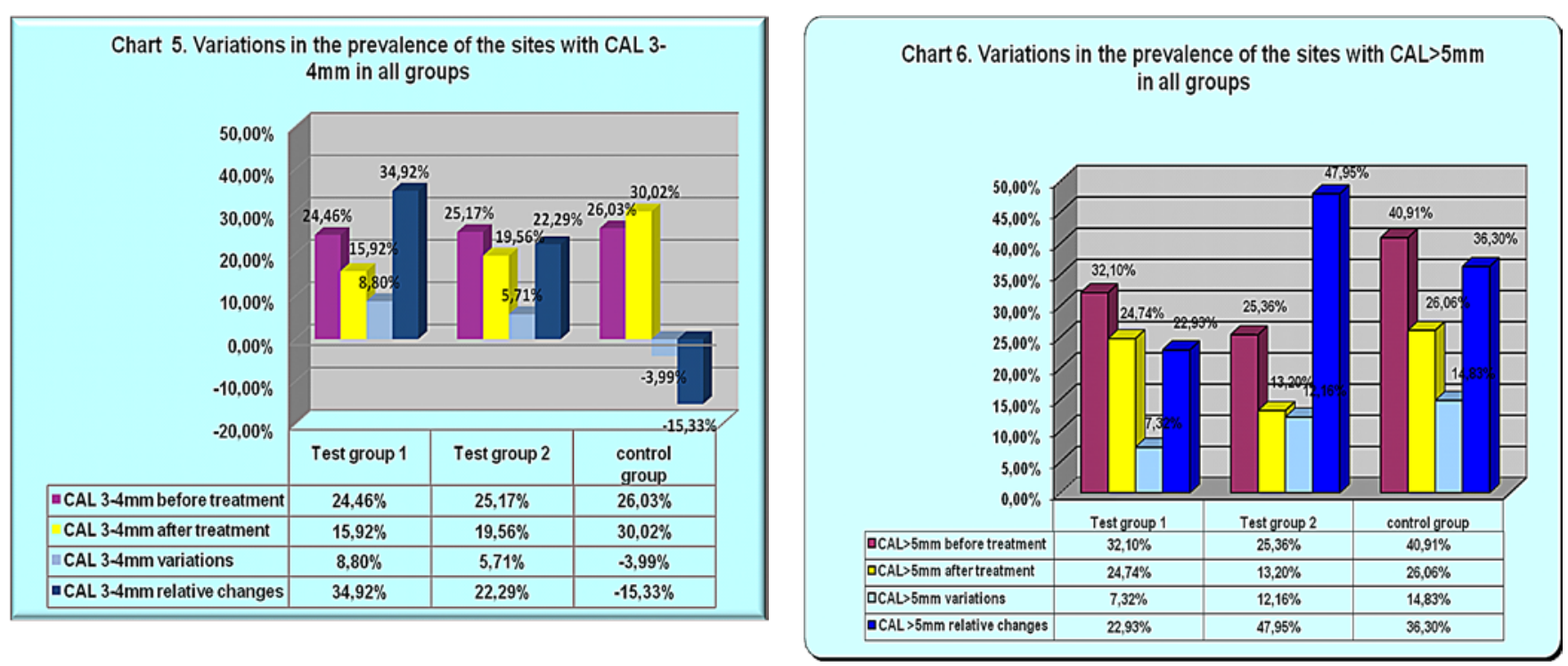

\section{CONCLUSION:}

The microbiological identification of the periodontal pathogens in deep periodontal pockets is recommended in periodontitis patients with moderate and severe periodontitis in order to target the periodontal treatment and to assure better control of the microbial factor resulting in better reduction

of the pocket depth and higher attachment gain. This approach could assist in achieving stable periodontal status, limitation of the requirements of surgical procedures thus leading to better maintenance of the periodontal health.

\section{REFERENCES:}

1. Armitage GC. The complete periodontal examination. Periodontol2000, 2004; 34: 22-33.

2. Djemileva, T. Periodontal diseases. Azer Sofia, 1999, 367p.

3. Haffajee AD, Socransky SS, Lindhe J, Kent RL, Okamoto H, Yoneyama T. Clinical risk indicators for periodontal attachment loss. J Clin Periodontol. 1991; 18(2):117-25.

4. Haffajee AD., Cugini MA., Dibart S., Smith C., Kent RL., Socransky SS. The effect of SRP on the clinical and microbiological parameters of periodontal disease. J Clin Periodontol 1997; 24: 324334.

5. Haffajee AD., Socransky SS. Microbiology of periodontal diseases: introduction. Periodontology 2000, 2005; 38: 9-12.

6. Holt SC., Ebersole JL. Porphyromonas gingivalis, Treponema denticola, and Tannerella forsythia: the "red complex", a prototype polybacterial pathogenic consortium in periodontitis. Periodontology 2000, 2005; 38: 72-122.

7. Loomer P. Microbiological diagnosis testing in the treatment of periodontal diseases. Periodontology 2000, Vol. 34, 2004, 49-56.

8. Mombelli A., McNabb H., Lang N. Black- pigmenting gram- negative bacteria in periodontal disease. II Screening strategies for detection of Porphyromonas gingivalis. J Periodontal Res 1991; 26: 308- 313.

9. Popova Chr., K. Kotsilkov. Effectiveness of the additional antimicrobial treatment in comparison with mechanical cause-related therapy of chronic periodontitis. Zabolekarski pregled 1/2007 70,71p.

10. Slots J., Listgarten MA. Bacteroides gingivalis, Bacteroides intermedius and Actinobacillus actinomycetemcomitans in human periodontal diseases. J Clin
Periodontol 1988; 15: 85-93.

11. Slots, J., Reynolds, H.S. \& Genco, R. J. (1980). Actinobacillus actinomycetemcomitans in human periodontal disease: a cross-sectional microbiological investigation. Infection and Immunity29, 1013-1020.

12. Socransky SS., Haffajee AD. Dental biofilms: difficult therapeutic targets. Periodontology 2000, 2002; 28: 12-55.

13. Socransky SS., Haffajee AD. Microbiology of periodontal disease. In: Lindhe J. Clinical periodontology and implant dentistry. 4 ed. 2003, 106- 149.

14. Van Winkelhoff A., Winkel E. Microbiological diagnostics in periodontics: biological significance and clinical validity. Periodontol 2000, 2005; 39: 40- 52.

15. Walker C. B., Karpinia K. \& Baehni P. Chemotherapeutics: antibiotics and other antimicrobials. Periodontology 2000; 36, 2004, 146-165 